九州大学学術情報リポジトリ

Kyushu University Institutional Repository

\title{
Multidimensional inversion of loop-loop frequency-domain EM data for resistivity and magnetic susceptibility
}

\section{Sasaki, Yutaka}

Kyushu University, Department of Earth Resources Engineering

Kim, Jung-Ho

Korea Institute of Geoscience and Mineral Resources, Exploration Geophysics \& Mining Engineering

Cho, Seong-Jun

Korea Institute of Geoscience and Mineral Resources, Exploration Geophysics \& Mining Engineering

http://hdl. handle. net/2324/25556

出版情報 : Geophysics. 75 (6)，pp.F213-F223，2010-12-10. Society of Exploration Geophysicists バージョン：

権利関係: (C) 2010 Society of Exploration Geophysicists. 


\title{
Multidimensional inversion of loop-loop frequency-domain EM data for resistivity and magnetic susceptibility
}

\author{
Yutaka Sasaki ${ }^{1}$, Jung-Ho Kim², and Seong-Jun $\mathrm{Cho}^{2}$
}

\begin{abstract}
Electromagnetic (EM) induction measurements are affected by resistivity and magnetic susceptibility. Thus, inverting EM data for resistivity alone can give misleading models if susceptible effects are strong. An inversion algorithm is presented to simultaneously recover multidimensional distributions of resistivity and susceptibility from various types of loop-loop frequencydomain EM data. The algorithm adopts a staggered-grid finitedifference method for the 3D forward solutions and computes the sensitivities with respect to resistivity and susceptibility from the forward solutions using the reciprocity principle. The algorithm is tested on synthetic data sets from ground-based small-loop, airborne, and Slingram EM surveys. It is shown that the simultaneous inversion of the small-loop EM data collected at a single
\end{abstract}

height is unstable and likely to produce unreliable susceptibility models because the effect of susceptibility is nearly independent of the frequency. However, if the data are obtained for multiple heights or different loop configurations, simultaneous inversion can produce more reliable susceptibility and resistivity models even if the data are contaminated by offset errors. It is also shown that although the simultaneous inversion of airborne EM data is relatively stable, adding data obtained at different heights helps to increase the reliability of the resistivity and susceptibility models. Among the loop-loop EM methods discussed here, the Slingram method is relatively insensitive to susceptibility anomalies and thus cannot be used to recover the susceptibility distribution via inversion even if the data are obtained using different loop configurations.

\section{INTRODUCTION}

Loop-loop frequency-domain electromagnetic (EM) methods use two loops (or coils) to generate EM fields at selected frequencies and detect the resulting secondary magnetic field, which is a diagnostic for deriving the electrical properties of the earth. The loop separation is usually sufficiently large compared with the loop radius that the transmitter loop can be regarded as a magnetic dipole. Depending on the loop-loop geometry, one can measure the different components of the earth's response to a vertical or horizontal magnetic dipole source. Several types of loop-loop EM systems are available for use in airborne (Palacky and West, 1991), marine (Chave et al., 1991), and ground-based surveys (Frischknecht et al., 1991).

Loop-loop EM data have been commonly interpreted in terms of resistivity under the assumption that the magnetic susceptibility is the same as that of free space. However, as shown in many studies (e.g., Beard and Nyquist, 1998; Hansen et al., 2005), the susceptibility of some earth materials is much greater than that of free space, and it is well recognized that the EM measurements can be affected by resistivity and susceptibility. Thus, interpretation of loop-loop EM data that accounts for susceptibility is not new. Successful applications include hazardous waste site characterization (Beard and Nyquist, 1998), geological mapping with higher spatial resolution than the magnetic data (Huang and Fraser, 2000), delineation of magnetically susceptible massive sulfide deposits (Farquharson et al. 2003), and locating buried building foundations and abandoned pipelines (Won and Huang, 2004).

There have been several attempts to perform 1D inversion of airborne loop-loop EM data for resistivity and susceptibility. Zhang and Oldenburg (1999) proposed a method to simultaneously recover $1 \mathrm{D}$ distributions of conductivity (or the reciprocal of resistivity) and susceptibility from horizontal loop data. Huang and Fraser (2003) inverted horizontal loop data to obtain the resistivity, susceptibility, and thickness of a 1D model. Farquharson et al. (2003) presented an inversion algorithm that constructs $1 \mathrm{D}$ conductivity and susceptibility models, automatically estimating the trade-off parameter using

Manuscript received by the Editor 7 January 2010; revised manuscript received 6 April 2010; published online 10 December 2010

${ }^{1}$ Kyushu University, Department of Earth Resources Engineering, Fukuoka, Japan. E-mail: sasaki@ mine.kyushu-u.ac.jp.

${ }^{2}$ Korea Institute of Geoscience and Mineral Resources, Exploration Geophysics \& Mining Engineering Department, Daejeon, Republic of Korea. E-mail: jungho@kigam.re.kr;mac@kigam.re.kr.

(C) 2010 Society of Exploration Geophysicists. All rights reserved. 
the generalized crossvalidation criterion. Such simultaneous inversions are expected to provide not only additional useful information (i.e., susceptibility) but also reliable resistivity models that are not affected by the susceptibility effect if the actual subsurface properties are locally uniform in the horizontal direction.

In this paper, we develop and test an inversion algorithm that can simultaneously produce multidimensional structures of resistivity and susceptibility from different types of loop-loop EM data. The forward modeling used in the inversion is based on a staggered-grid finite-difference method, and the sensitivities of the data with respect to resistivity and susceptibility are obtained from the forward modeling solutions by applying the reciprocity principle. We show through synthetic examples the usefulness of collecting data from multiple heights and/or different loop configurations to reduce the nonuniqueness of the simultaneous inversion.

\section{FORWARD MODELING}

At the frequencies used in many EM methods, displacement currents are negligible. Assuming time dependence of $e^{i \omega t}$ and including varying permeability, we obtain the differential equation for the electric field from Maxwell's equations as

$$
\nabla \times \frac{\mu_{0}}{\mu} \nabla \times \mathbf{E}+i \omega \mu_{0} \sigma \mathbf{E}=-i \omega \mu_{0} \mathbf{J}_{s} .
$$

In this equation, $\omega$ is the angular frequency, $\sigma$ is the conductivity, $\mu$ is the permeability, and $\mu_{0}$ is the permeability of free space $\left(4 \pi 10^{-7} \mathrm{H} / \mathrm{m}\right)$. The impressed source is denoted $\mathbf{J}_{s}$, and is specified depending on whether the equation is formulated in terms of the total or secondary field. In a secondary-field formulation, $\mathbf{E}$ is replaced by $\mathbf{E}_{s}$ and the source vector becomes an equivalent source (Alumbaugh et al., 1996) defined by

$$
\mathbf{J}_{s}=\left(\sigma-\sigma_{p}\right) \mathbf{E}_{p}+\nabla \times\left[\left(\frac{\mu-\mu_{0}}{\mu}\right) \mathbf{H}_{p}\right],
$$

where $\mathbf{E}_{p}$ and $\mathbf{H}_{p}$ are the background electric and magnetic fields, respectively, and $\sigma_{p}$ is the background conductivity of a uniform halfspace having the free-space permeability. The magnetic field above the earth's surface can be obtained through

$$
\mathbf{H}=-\frac{1}{i \omega \mu_{0}} \nabla \times \mathbf{E} .
$$

In Yee's (1966) finite-difference (FD) scheme using a staggered grid, the model (including an air layer) is discretized into rectangular cells. Applying the FD approximation to equation 1 and using the Dirichlet boundary condition yields a linear system of equations

$$
\left(\mathbf{A}+i \omega \mu_{0} \mathbf{D}\right) \mathbf{f}=-i \omega \mu_{0} \mathbf{s},
$$

where $\mathbf{f}$ is the unknown vector for the electric field and $\mathbf{s}$ is the source vector. The FD representation of equation 1 for the secondary electric field was presented by Alumbaugh et al. (1996). The coefficient matrix in equation 4 is usually represented by a single matrix, but here it is separated into two parts, a real symmetric matrix $\mathbf{A}$ and a real diagonal matrix $\mathbf{D}$. The matrix $\mathbf{A}$ depends on the permeability and the grid spacing but not the conductivity, whereas $\mathbf{D}$ depends on the conductivity and grid spacing but not the permeability. This representation of the coefficient matrix is useful later when explaining how to derive the sensitivity of the EM response with respect to the conductivity and permeability.

The solution to equation 4 can be obtained using the biconjugate gradient (BCG) method. As a preconditioner, an incomplete Cholesky decomposition is applied partially, only to the diagonal sub-blocks of the coefficient matrix that are positive definite (Mackie et al., 1994). Furthermore, to improve the convergence rates at low frequencies, the preconditioned BCG method is alternated with a procedure designed to enforce a static divergence-free condition on the current density (Smith, 1996).

\section{SENSITIVITY}

The sensitivities of the EM responses are commonly derived from the integral or differential form of Maxwell's equations using the adjoint equation approach (e.g., Weidelt, 1975; McGillivray et al., 1994). There is an alternative approach that starts with the discrete form of a differential equation, such as equation 4 (e.g., Tripp et al. 1984; Sasaki, 2001).

Although equation 4 is actually solved for the secondary field to eliminate the need to approximate the rapid variation of the primary field in the vicinity of the source, let us assume here that the unknown vector denotes the total field to illustrate how to derive the sensitivity. Because the source vector is independent of the conductivity and permeability, differentiating both sides of equation 4 with respect to the conductivity of a particular block $\sigma_{m}$ yields

$$
\left(\mathbf{A}+i \omega \mu_{0} \mathbf{D}\right) \frac{\partial \mathbf{f}}{\partial \sigma_{m}}=-i \omega \mu_{0} \frac{\partial \mathbf{D}}{\partial \sigma_{m}} \mathbf{f} .
$$

Likewise, differentiating equation 4 with respect to the permeability $\mu_{m}$, we have

$$
\left(\mathbf{A}+i \omega \mu_{0} \mathbf{D}\right) \frac{\partial \mathbf{f}}{\partial \mu_{m}}=-\frac{\partial \mathbf{A}}{\partial \mu_{m}} \mathbf{f}=-i \omega \mu_{0}\left(\frac{1}{i \omega \mu_{0}} \frac{\partial \mathbf{A}}{\partial \mu_{m}} \mathbf{f}\right)
$$

By analogy with equation 4, the derivatives of the electric field with respect to $\sigma_{m}$ and $\mu_{m}$ can be interpreted as the responses to a collection of electric dipole sources defined by the right-hand side of equations 5 and 6 , respectively. Note that these current sources are present only within the block of interest. Using the reciprocity, it follows that the derivative of the electric field at a receiver point is the weighted sum of the electric fields within the block due to a unit electric dipole source placed at the receiver point. The weights in the summation are defined by the right-hand side of equation 5 or 6 .

The magnetic field is given by the curl of the electric field, as shown in equation 3 , and thus can be approximated as a linear combination of the electric fields at the neighboring points on the grid. Likewise, the magnetic dipole source at the same location is expressed with the same linear combination of the electric dipole sources but without the scaling factor $\left(i \omega \mu_{0}\right)^{-1}$. Thus, the procedure for obtaining the derivatives of the electric field can also be used to obtain the derivatives of the magnetic field if the weighted sum within the block is multiplied by the scaling factor $\left(i \omega \mu_{0}\right)^{-1}$, which is required when the magnetic field at the receiver point is replaced with the unit magnetic dipole source.

In the inversion examples shown later, we invert EM data for the logarithm of resistivity and the susceptibility. The sensitivity of the magnetic field for resistivity is given by 


$$
\frac{\partial \mathbf{H}}{\partial \ln \rho_{m}}=-\sigma_{m} \frac{\partial \mathbf{H}}{\partial \sigma_{\mathbf{m}}},
$$

where $\rho_{m}$ is the resistivity. The permeability is related to the susceptibility $\kappa$ through

$$
\mu=\mu_{0}(1+\kappa) .
$$

Thus, the sensitivity for susceptibility can be obtained by using

$$
\frac{\partial \mathbf{H}}{\partial \kappa}=\mu_{0} \frac{\partial \mathbf{H}}{\partial \mu} .
$$

\section{INVERSION METHOD}

The data used in the inversion are secondary magnetic fields normalized by the free-space field. The in-phase and quadrature components for any particular frequency and loop-loop geometry are considered as independent data. The model region is divided into a set of rectangular blocks. The resistivity and susceptibility are assumed to be constant in each block.

The inverse problem is nonlinear and requires an iterative procedure. Let $\Delta \mathbf{m}_{\rho}$ and $\Delta \mathbf{m}_{\kappa}$ be the perturbations for the resistivity and susceptibility parts of the starting (or current) model, $\mathbf{m}_{\rho}^{(n-1)}$ and $\mathbf{m}_{\kappa}^{(n-1)}$, respectively. The model parameters used in the inversion are the logarithm of resistivity and the susceptibility transformed by using

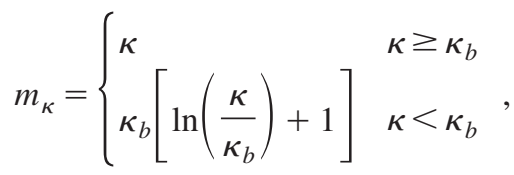

where $\kappa_{b}$ is set to 0.01 in SI units. The above transformation ensures that the susceptibilities in the constructed model are positive (Zhang and Oldenburg, 1999). The reason for not using the logarithm of susceptibility is that it tends to cause the model to become excessively rough as the magnitude increases.

The objective function to be minimized is

$$
\begin{aligned}
\phi= & \left\|\mathbf{W}\left[\Delta \mathbf{d}-\mathbf{J}_{\rho} \Delta \mathbf{m}_{\rho}-\mathbf{J}_{\kappa} \Delta \mathbf{m}_{\kappa}\right]\right\|^{2}+\lambda^{2} \| \mathbf{C}\left[\mathbf{m}_{\rho}^{(n-1)}\right. \\
& \left.+\Delta \mathbf{m}_{\rho}\right]\left\|^{2}+\lambda^{2} \alpha^{2}\right\| \mathbf{C}\left[\mathbf{m}_{\kappa}^{(n-1)}+\Delta \mathbf{m}_{\kappa}\right] \|^{2},
\end{aligned}
$$

where $\mathbf{J}_{\rho}$ and $\mathbf{J}_{\kappa}$ are the sensitivity matrices with respect to the model parameters related to the resistivities and susceptibilities, respectively. The matrix $\mathbf{W}$ is a diagonal matrix that assigns weights to each datum, $\mathbf{C}$ is a second-order FD operator, and $\Delta \mathbf{d}$ is the vector of differences between the observed and predicted data. The parameter $\lambda$ is a Lagrange multiplier, and $\alpha$ controls the relative importance of the smoothness constraints on the resistivity and susceptibility models. The minimization of $\phi$ is equivalent to obtaining the leastsquares solution of the rectangular system of equations

$$
\left[\begin{array}{cc}
\mathbf{W J}_{\rho} & \mathbf{W} \mathbf{J}_{\kappa} \\
\lambda \mathbf{C} & \mathbf{0} \\
\mathbf{0} & \lambda \alpha \mathbf{C}
\end{array}\right]\left\{\begin{array}{l}
\Delta \mathbf{m}_{\rho} \\
\Delta \mathbf{m}_{\kappa}
\end{array}\right\}=\left\{\begin{array}{l}
\mathbf{W} \Delta \mathbf{d} \\
-\lambda \mathbf{C m}_{\rho}^{(n-1)} \\
-\lambda \alpha \mathbf{C m}_{\kappa}^{(n-1)}
\end{array}\right\} .
$$

We use the modified Gram-Schmidt method (Bjorck, 1996) to solve equation 12 .

\section{VERIFICATION OF FORWARD SOLUTION}

To verify the accuracy of the FD solution for loop-loop EM simulations, we compared it with the theoretical solution for a three-layer model (Figure 1a). The 1D model consists of an anomalous layer with resistivity of $10 \mathrm{ohm}-\mathrm{m}$ and susceptibility of 0.05 (SI units) buried $1 \mathrm{~m}$ deep in a half-space with resistivity of $100 \mathrm{ohm}-\mathrm{m}$ and zero susceptibility. The thickness of the middle layer is $3 \mathrm{~m}$. A vertical magnetic dipole is located $1 \mathrm{~m}$ above the earth's surface, and a receiver that is located $2 \mathrm{~m}$ apart and at the same height as the source samples the vertical component of the magnetic field at frequencies between 2500 and 20,000 Hz. In the 3D modeling, the earth and air were divided into $103 \times 39 \times 35$ cells in the $x, y$, and $z$ coordinate directions. The smallest cell size used was $0.5 \times 0.5 \times 0.25 \mathrm{~m}$, and the largest cell size was $32 \times 32 \times 32 \mathrm{~m}$. The comparison between the numerical and 1D solutions is shown in Figure 1b, where the real (inphase) and imaginary (quadrature) components of the secondary magnetic fields are plotted in parts per million ( $\mathrm{ppm}$ ) of the freespace field. The agreement is excellent, with a maximum relative error of $1.1 \%$ observed for the real component at $2500 \mathrm{~Hz}$. As an example, Figure 2 shows the convergence rate of the FD solution at $10,000 \mathrm{~Hz}$. The squared residual norm is defined as

$$
\left\|\mathbf{K f}-\mathbf{s}^{\prime}\right\|^{2} /\left\|\mathbf{s}^{\prime}\right\|^{2}
$$

where $\mathbf{K}$ and $\mathbf{s}^{\prime}$ represent the coefficient matrix and right-hand side in equation 4, respectively. The numerical results in Figure $1 \mathrm{~b}$ were obtained when the stopping criterion was set to $10^{-6}$ and are practically the same as when the criterion $10^{-8}$ was used. The average

a)

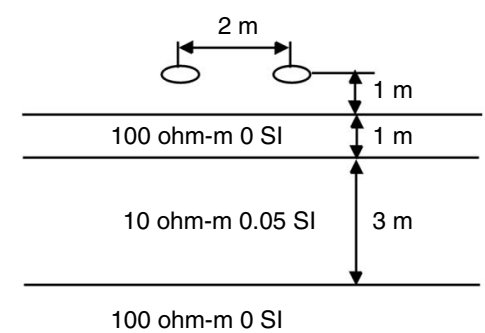

b)

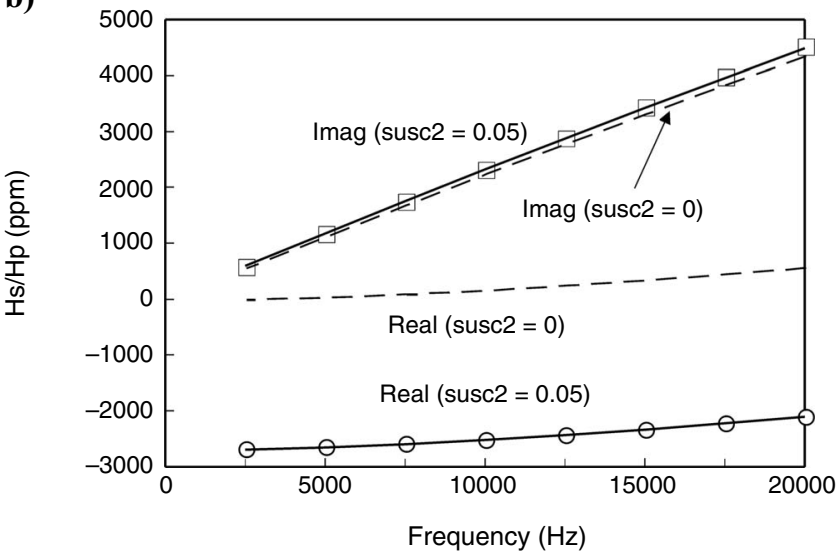

Figure 1. (a) Loop-loop configuration over a three-layer model. The source is a vertical magnetic dipole, and the receiver samples the vertical component of the secondary magnetic field. (b) Comparisons of the FD (symbols) and theoretical (solid lines) solutions. The dashed lines represent the response for the 1D model that is the same as the one in (a) except that the second layer is nonsusceptible. 
computation time per one frequency was $33 \mathrm{~s}$ on a personal computer (PC) with a $2.50-\mathrm{GHz}$ Core 2 processor.

\section{SYNTHETIC DATA EXAMPLES}

We tested our simultaneous inversion algorithm on synthetic data sets obtained for three different loop-loop EM methods. In the first example, we assume that data are collected using a hand-held EM instrument in which the transmitter and receiver loops are rigidly fixed (e.g., Won, 2003); we refer to this type of data as small-loop EM data. The second example involves a helicopter towed-bird EM system that maintains precise loop separation. The final example is obtained from the so-called Slingram method (Frischknecht et al., 1991), which uses separate transmitter and receiver loops connected by a reference cable. The loop configurations considered here are horizontal coplanar (HCP), vertical coplanar (VCP), and vertical coaxial (VCX) systems, as shown in Figure 3.

\section{Small-loop EM data}

The model used to generate small-loop EM data is shown in Figure 4. This model is composed of three anomalous rectangular bod-

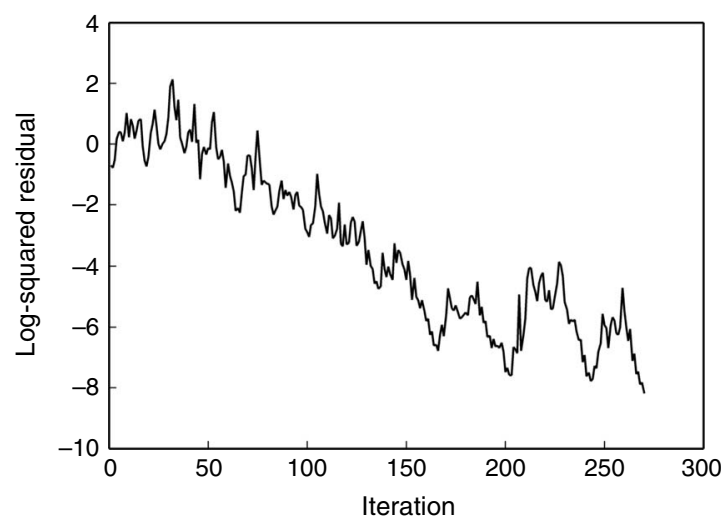

Figure 2. Plot of the normalized squared residual against iteration number for the FD solution at 10,000 $\mathrm{Hz}$ for the 1D model shown in Figure 1.

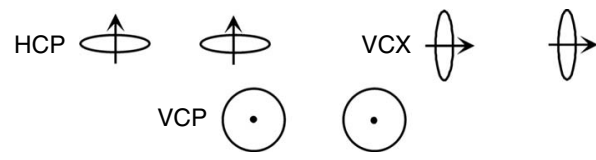

Figure 3. Geometries of typical loop-loop systems: the HCP, VCP, and VCX configurations.

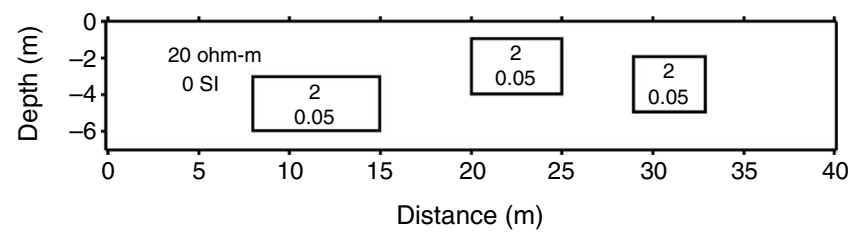

Figure 4. 2D model used to generate the synthetic data for groundbased small-loop EM surveys. The model consists of three buried blocks of 2-ohm-m resistivity and 0.05 (SI units) susceptibility in a nonsusceptible 20-ohm-m background half-space. ies of 2-ohm-m resistivity and 0.05 (SI units) susceptibility embedded in a uniform half-space of 20 -ohm-m resistivity and zero susceptibility. The transmitter and receiver loops are assumed to be $2 \mathrm{~m}$ apart and positioned $1 \mathrm{~m}$ above the earth's surface. The loop-loop geometry has the HCP configuration; that is, data are of the vertical component of the secondary magnetic field generated by a vertical magnetic dipole source. The frequencies used in this inversion experiment are 5000, 10,000, 20,000, and 40,000 Hz. The magnetic field responses were computed using a $103 \times 39 \times 35$ cell grid for 39 locations at intervals of $1 \mathrm{~m}$. The HCP responses for the model in Figure 4, along with those for the model having the same resistivity distribution but zero susceptibility, are shown in Figure 5a and b, where the secondary magnetic fields are plotted in ppm of the freespace field. It is clear from Figure 5a that the effect of susceptibility on the quadrature components is negligible. In contrast, the susceptibility anomaly causes significant differences in the in-phase responses (Figure 5b), particularly over the shallowest (central) susceptibility anomaly, resulting in negative responses at lower frequencies. The differences in the in-phase responses between the models with and without susceptibility anomalies (Figure 5c) show that for the given frequency range the effect of susceptibility on the in-phase response is nearly independent of the frequency and hence manifests itself as static shifts from the responses of the nonsusceptible model. Note that such downward static shifts depend on the susceptibility but not the resistivity for the given frequency range.

We inverted the HCP data at the four frequencies for resistivity and susceptibility. Before the inversion, the data were contaminated with Gaussian noise with a standard deviation of $1 \%$ of the amplitude of the datum, except if the noise was less than $30 \mathrm{ppm}$, in which case a standard deviation of $30 \mathrm{ppm}$ was used. In this and the following inversions, the model region was divided into $280(40 \times 1 \times 7)$ blocks of unknown parameters, and the forward calculations used a grid of $63 \times 29 \times 27$ cells. The parameters $\lambda$ and $\alpha$ were fixed at 0.2 and 10 , respectively, throughout the inversion. The starting model was a homogeneous half-space of 50-ohm-m resistivity and $10^{-4}$ (SI units) susceptibility.

Figure $6 \mathrm{a}$ and $\mathrm{b}$ shows the resistivity and susceptibility models obtained at the sixth iteration, respectively. The recovered resistivity represents the true model reasonably well. In contrast, the susceptibility is poorly resolved except for the central body. The data misfit after six iterations is $180 \mathrm{ppm}$, which is far larger than the true misfit of $76 \mathrm{ppm}$; further iterations do not reduce the misfit significantly. Such a poor data fit is attributed solely to the poor fit to the in-phase components and hence the poor reconstruction of susceptibility. When different values of $\alpha$ were used, the result of inversions (not shown) was that the susceptibility models were considerably different but the resistivity models were almost identical to that seen in Figure 6a. This feature is consistent with results from 1D inversion studies (Zhang and Oldenburg, 1999; Farquharson et al., 2003), showing that the recovered resistivity is insensitive to the choice of the relative weights of the smoothing constraints on the resistivity and susceptibility models.

The above results indicate that the simultaneous inversion for resistivity and susceptibility is not stable for the given data (HCP data at a single height), although the resistivity model is recovered well. This is because the effect of susceptibility on the magnetic field response is virtually static and thus has no inherent depth resolution, like static magnetic data. This suggests that recovering the susceptibility from the EM data with such a characteristic requires changing the survey geometry rather than changing the transmitter frequency. 
For hand-held instruments having a fixed HCP configuration, the VCP data can be obtained by rotating the instrument along the survey line by $90^{\circ}$. The VCP response at a height of $1 \mathrm{~m}$ is shown in Fig- ure $5 \mathrm{~d}$ and $\mathrm{e}$. It is seen that the VCP data provide complementary information of the resistivity and susceptibility. Figure $6 \mathrm{c}$ and $\mathrm{d}$ shows the result of inverting the $\mathrm{HCP}$ and $\mathrm{VCP}$ data at the four frequencies. a)

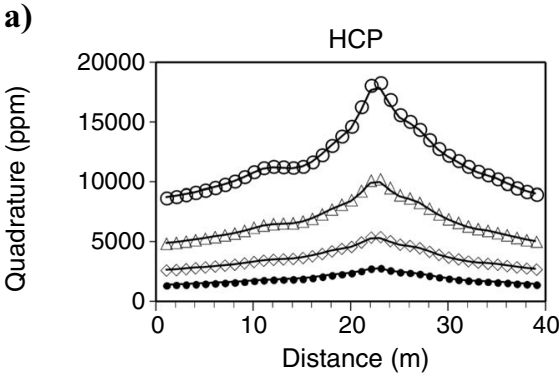

d)

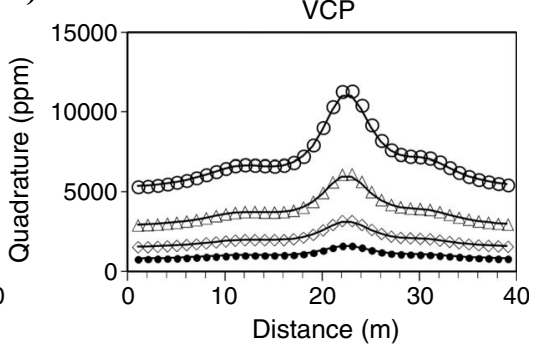

b)

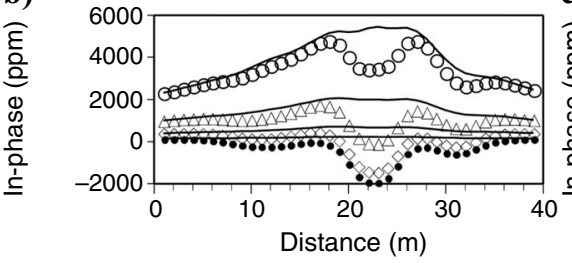

e)

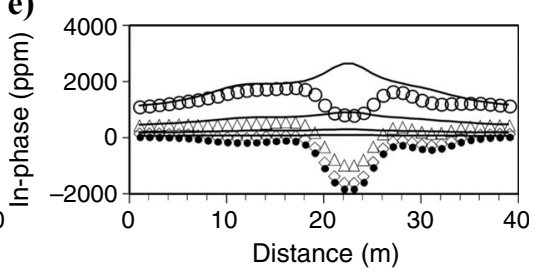

c)

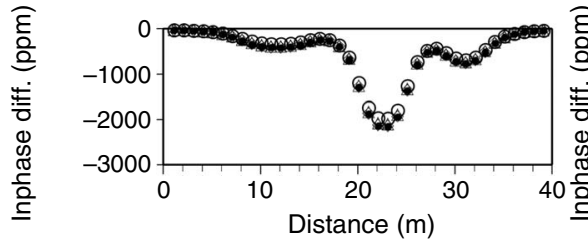

f)
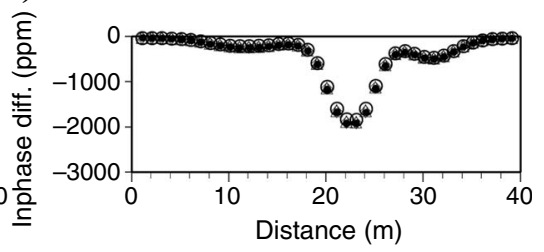

Figure 5. Small-loop EM responses for the HCP (left) and VCP (right) configurations, calculated for the model shown in Figure 4 (symbols) and the same model without susceptibility anomalies (solid lines). The top panels present the quadrature components. The middle panels show the in-phase components. The bottom panels show the differences in the in-phase responses between the models with and without susceptibility anomalies. The frequencies used are $40,000 \mathrm{~Hz}$ (open circles), $20,000 \mathrm{~Hz}$ (triangles), 10,000 Hz (diamonds), and $5000 \mathrm{~Hz}$ (solid circles). a)

-

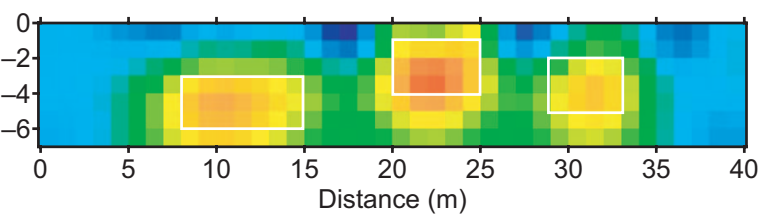

c)

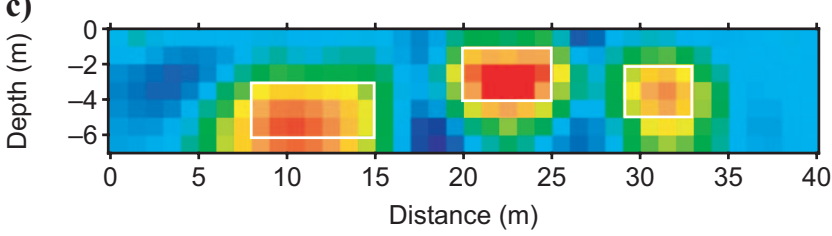

e)

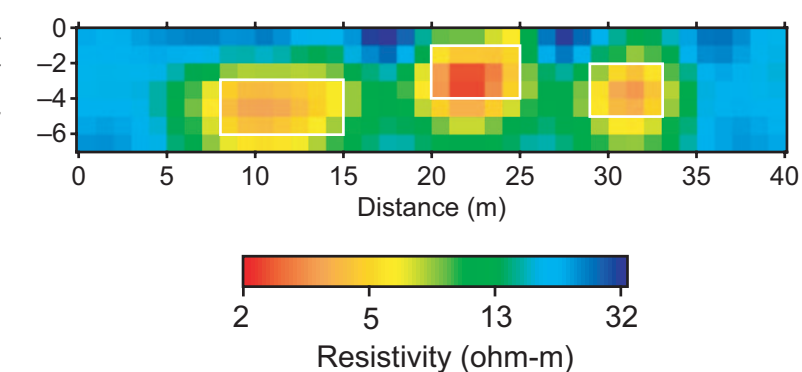

b)

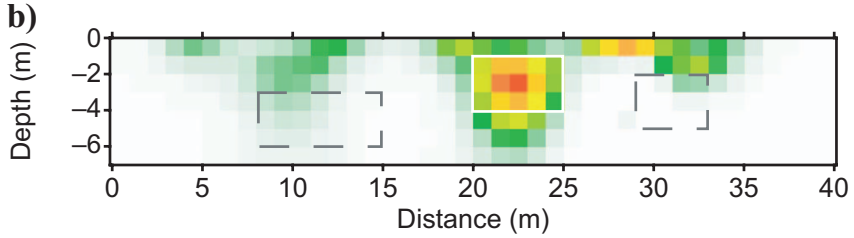

d)

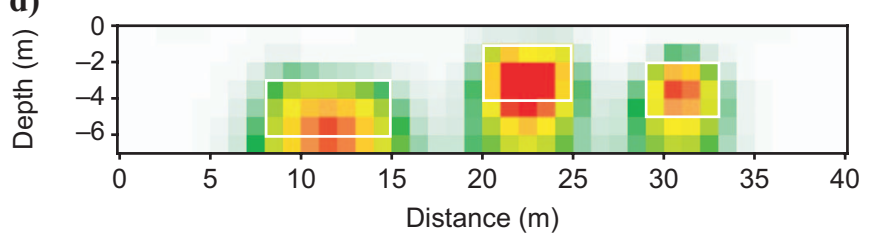

f)

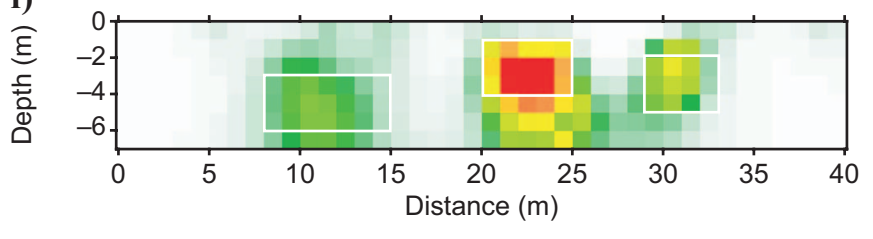

Figure 6. Resistivity (left) and susceptibility (right) models obtained from inverting three small-loop EM data sets for the model shown in Figure 4. The top, middle, and bottom panels are for the cases in which data are collected with the HCP configuration at a height of $1 \mathrm{~m}$, the HCP and VCP configurations at a height of $1 \mathrm{~m}$, and the HCP configuration at heights of 1 and $1.5 \mathrm{~m}$, respectively. 
The final data misfit was $73 \mathrm{ppm}$. The susceptibility model recovered from the HCP and VCP data is much improved, showing three isolated anomalies, and the resistivity model is better defined than that presented in Figure 6a.

Another way to change the survey geometry of the small-loop EM method is to change the height of the loop pair. Figure 6e and $\mathrm{f}$ shows the resistivity and susceptibility models, respectively, obtained from the inversion of the HCP data for heights of 1 and $1.5 \mathrm{~m}$. It is seen that the recovered susceptibility model is a fair improvement on the result for the HCP data for a single height (Figure 6b). From this synthetic example we see that collecting data from different loop-loop configurations or different heights is essential in simultaneously constructing the resistivity and susceptibility models from smallloop EM data.

\section{Small-loop EM data with offset errors}

One inherent problem related to the quality of the small-loop EM data is offset errors, which refer to the instrument response not being zero in free space. Sasaki et al. (2008) showed that if data are collected at multiple heights, it is possible to recover the resistivity well from the offset error-contaminated data by simultaneously inverting for resistivity and offset error. Here, we examine the performance of the simultaneous inversion for the resistivity, susceptibility, and offset error in the case in which data contain offset errors, as presented in Table 1, in addition to Gaussian noise. In the inversion, it is assumed that the offset errors for the in-phase and quadrature components at each frequency are constant during the course of data collection and independent of each other. Note that the offset errors remain the same even if the instrument is rotated or its height is changed. We consider three cases. In cases 1 and 2, HCP data are collected at two heights ( 1 and $1.5 \mathrm{~m})$ and three heights $(1,1.5$, and $2 \mathrm{~m})$, respectively. In case 3, HCP and VCP data are collected at two heights (1 and $1.5 \mathrm{~m}$ ). The frequencies used are the same as in the previous example.
Figure $7 \mathrm{a}$ and $\mathrm{b}$ shows the resistivity and susceptibility models, respectively, constructed from the simultaneous inversion of the HCP data for heights of 1 and $1.5 \mathrm{~m}$ (case 1). The starting models and other parameters were kept the same as in the previous example. Comparing this result with that obtained using the data without the offset errors (Figure 6e and f), we see that the simultaneous inversion does not recover the resistivity and susceptibility models well, particularly the deepest (leftmost) susceptibility anomaly, because of trade-off with the offset errors. The recovered offset errors, presented in Table 1, are roughly correct but are not good enough to define the resistivity and susceptibility well.

Figure $7 \mathrm{c}$ and $\mathrm{d}$ shows the result of the simultaneous inversion of the data for three heights, $1,1.5$, and $2 \mathrm{~m}$ (case 2). With the incorporation of additional data for the 2-m height, the offset errors are better determined (see Table 1), and hence the resistivity and susceptibility models are better recovered than in the case of using data for only two heights.

Figure $7 \mathrm{e}$ and $\mathrm{f}$ shows the result of simultaneous inversion of the $\mathrm{HCP}$ and VCP data for two heights, 1 and $1.5 \mathrm{~m}$ (case 3). The reconstructed resistivity and susceptibility models define the three anomalies well and are comparable to the result obtained from the data without the offset errors (Figure $6 \mathrm{c}$ and d). The estimated offset errors are the best among the three cases.

\begin{abstract}
Airborne EM data
Data were generated from the model shown in Figure 8. This model consists of four rectangular bodies of $10-\mathrm{ohm}-\mathrm{m}$ resistivity and 0.1 (SI units) susceptibility in a background medium of 1000-ohm $-\mathrm{m}$ resistivity and zero susceptibility. The loop separation is $8 \mathrm{~m}$ and the height of the loop pair is $30 \mathrm{~m}$. The HCP responses were computed at frequencies of $1000,2700,7400$, and $20,000 \mathrm{~Hz}$ along a 500 $-\mathrm{m}$ line at intervals of $10 \mathrm{~m}$. Figure $9 \mathrm{a}$ and $\mathrm{b}$ shows the HCP responses for the model and those for the model with the same resistivity but zero susceptibility. We see that the quadrature response (Figure 9a) is hardly affected by the susceptibility and that the susceptibility anomalies reduce the in-phase response (Figure 9b), causing the inphase response to become negative at lower frequencies over shal-
\end{abstract}

Table 1. Offset errors assigned to the in-phase (I) and quadrature (Q) components of the small-loop EM data at four frequencies and those recovered from simultaneous inversion. Cases 1 and 2 refer to the inversion results for the data sets obtained at two heights $(1$ and $1.5 \mathrm{~m})$ and three heights $(1,1.5$, and $2 \mathrm{~m})$, respectively. Case 3 is for the HCP and VCP data obtained at heights of 1 and $1.5 \mathrm{~m}$. The numerals in the parentheses show the difference between the estimated and true values

Offset error (ppm)

\begin{tabular}{|c|c|c|c|c|c|}
\hline Freq. (Hz) & Comp. & True & Case 1 & Case 2 & Case 3 \\
\hline 5000 & I & -1000 & $-875(125)$ & $-995 \quad(5)$ & $-1005(-5)$ \\
\hline 10000 & I & 1200 & 1286 & $1141(-59)$ & $1194(-6)$ \\
\hline 20000 & I & -2300 & -2189 & $-2400(-100)$ & $-2289 \quad(11)$ \\
\hline 40000 & I & -1400 & $-1056(344)$ & $-1401 \quad(-1)$ & $-1347 \quad(53)$ \\
\hline 5000 & Q & -2300 & $-2330(-30)$ & $-2379(-79)$ & $-2302(-2)$ \\
\hline 10000 & Q & 2650 & 2651 & $2572(-78)$ & $2652 \quad(2)$ \\
\hline 20000 & Q & 3800 & $3902(102)$ & $3790(-10)$ & $3802 \quad$ (2) \\
\hline 40000 & $\mathrm{Q}$ & -3900 & $-3744(156)$ & $-3842 \quad(58)$ & $-3919(-19)$ \\
\hline
\end{tabular}


a)

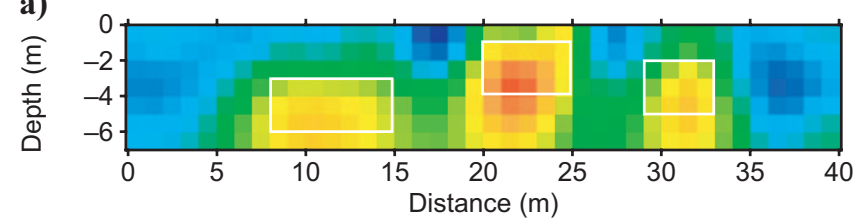

c)

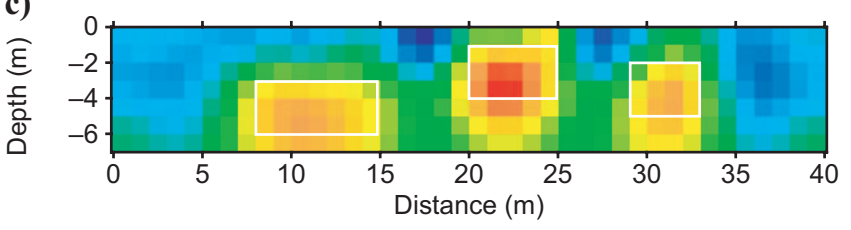

e)
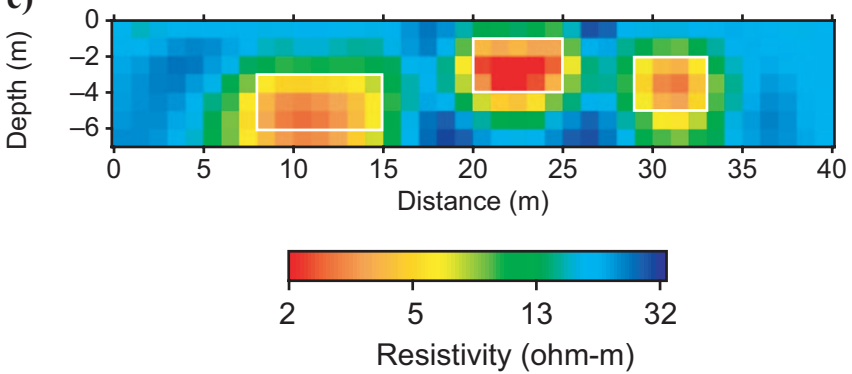

b)

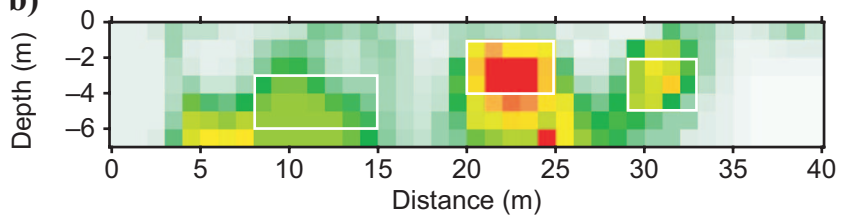

d)

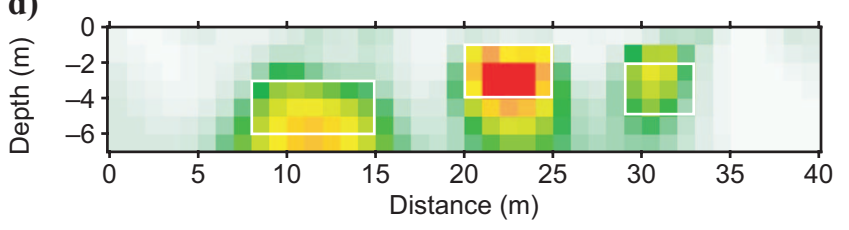

f)
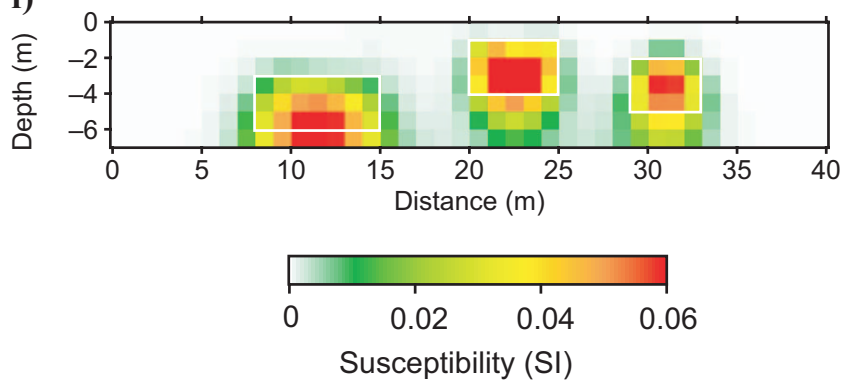

Figure 7. Resistivity (left) and susceptibility (right) models obtained from simultaneous inversion of the offset error-contaminated data for the model shown in Figure 4. The top and middle panels are for the cases in which the HCP data are collected at two heights (1 and $1.5 \mathrm{~m})$ and three heights $(1,1.5$, and $2 \mathrm{~m})$, respectively. The bottom panels are for the case in which the HCP and VCP data are collected at heights of 1 and $1.5 \mathrm{~m}$.

lower anomalies. Furthermore, as shown in Figure 9c, the effect of susceptibility on the in-phase response varies with the frequency at higher frequencies and becomes almost the same at frequencies of 1000 and $2700 \mathrm{~Hz}$.

We carried out the simultaneous inversion of the data shown in Figure $9 \mathrm{a}$ and $\mathrm{b}$. In the inversions, the model region was divided into $416(52 \times 1 \times 8)$ blocks, and a grid of $69 \times 29 \times 34$ was used in the forward calculations. The standard deviation of the noise added to each datum was $1 \%$ of the amplitude of the datum, except if the noise was less than $2 \mathrm{ppm}$, in which case a standard deviation of $2 \mathrm{ppm}$ was used. The starting model was a half-space of 200-ohm-m resistivity and $10^{-4}$ (SI units) susceptibility. The parameters $\lambda$ and $\alpha$ used were the same as those in the small-loop EM examples. Figure 10a and $\mathrm{b}$ shows the resistivity and susceptibility models obtained at the sixth iteration, respectively. The final data misfit was $11 \mathrm{ppm}$. The recovered resistivity model (Figure 10a) is a poor representation of the true model and even worse than the susceptibility model in terms of the lateral resolution. Note that false conductive anomalies appear between the actual anomalies.

The reason for such poor resolution of the resistivity is that the footprint size, a measure of the lateral resolution of an airborne EM system (e.g., Reid et al., 2006), is too large at a height of $30 \mathrm{~m}$ to resolve anomalous bodies of the given sizes. As shown in Figure 9d and e, the profiles of the HCP response have more distinct lateral variations when the height of the loop pair was lowered from 30 to $20 \mathrm{~m}$. Figure 10c and d shows the result of the simultaneous inversion of the data for the reduced height $(20 \mathrm{~m})$. As expected, the resistivity model is much better recovered, and the susceptibility is comparable to that for a height of $30 \mathrm{~m}$. The final data misfit was $15 \mathrm{ppm}$. The simultaneous inversion of the airborne data for single heights is stable compared with that of ground-based small-loop

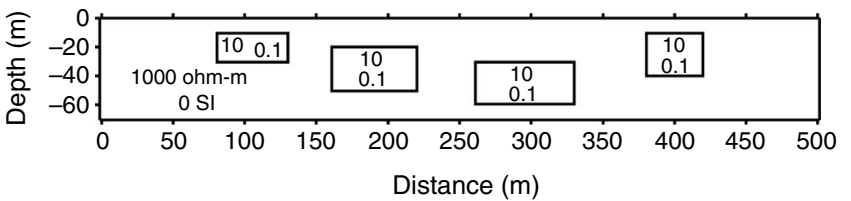

Figure 8. 2D model used to generate the synthetic data for airborne EM surveys. The model consists of four buried blocks of 10-ohm-m resistivity and 0.1 (SI units) susceptibility in a nonsusceptible 1000ohm-m background half-space.

data. The reason for this is that the airborne data are affected by the susceptibility anomalies mainly at lower frequencies because of the much greater height of the loops above the susceptibility anomalies.

Figure 10e and $\mathrm{f}$ shows the result of simultaneous inversion when both data sets (for heights of 20 and $30 \mathrm{~m}$ ) were used. The final data misfit was $8 \mathrm{ppm}$. Although the recovered resistivity model (Figure $10 \mathrm{e}$ ) is almost the same as that for measurements at a 20-m height (Figure 10c), the susceptibility model (Figure 10f) shows four anomalous zones more clearly than did those for single-height measurements. The poor resolution for the lower boundaries of the susceptibility anomalies may reflect the inherent limitation of depth resolution arising from the fact that the susceptibility effects on airborne EM data are inversely proportional to the cube of the loop height (Won and Huang, 2004).

\section{Slingram EM data}

A 2D model, shown in Figure 11, consists of an elongated, depressed conductive body embedded in a half-space of 200-ohm-m resistivity and zero susceptibility. This body has a resistivity of 
Figure 9. Airborne HCP responses for heights of $30 \mathrm{~m}$ (left) and $20 \mathrm{~m}$ (right) calculated for the model shown in Figure 8 (symbols) and the same model without susceptibility anomalies (solid lines). The top and middle panels show the quadrature and inphase components, respectively. The bottom panels show the differences in the in-phase responses between the models with and without susceptibility anomalies. The frequencies used are $20,000 \mathrm{~Hz}$ (open circles), $7400 \mathrm{~Hz}$ (triangles), $2700 \mathrm{~Hz}$ (diamonds), and $1000 \mathrm{~Hz}$ (solid circles).
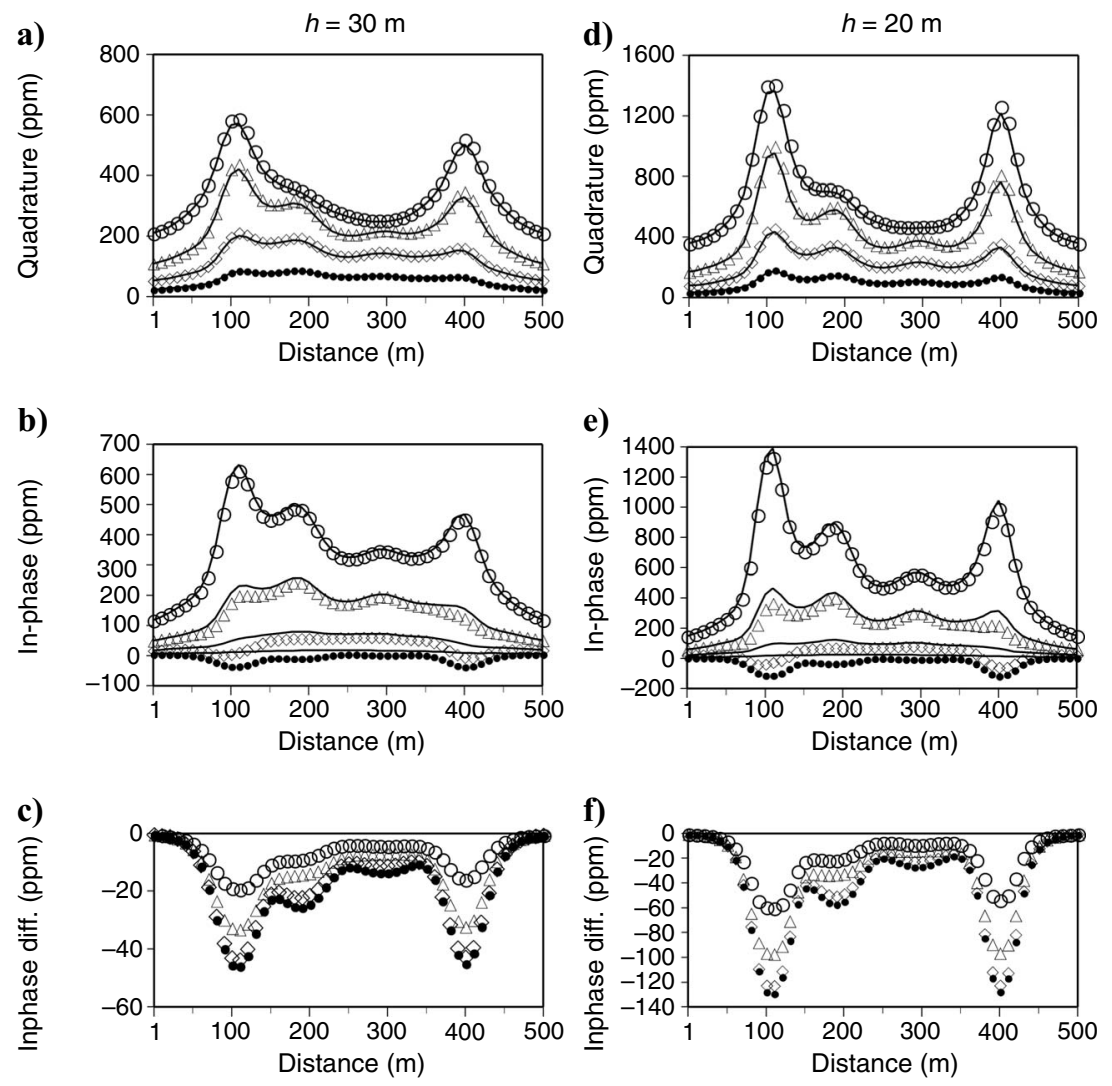

a)

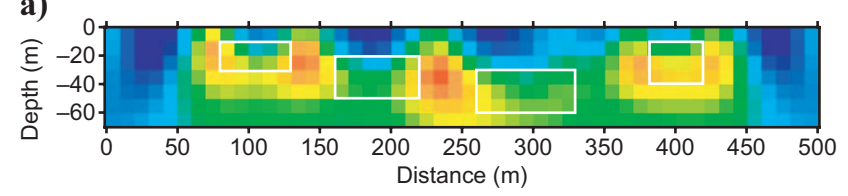

c)

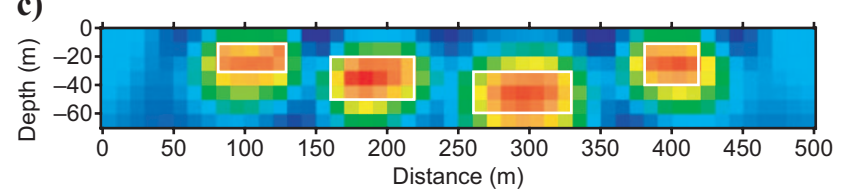

e)

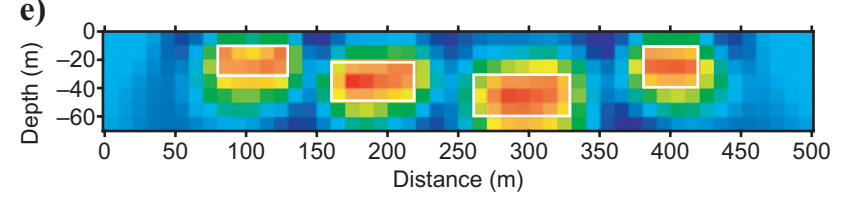

b)

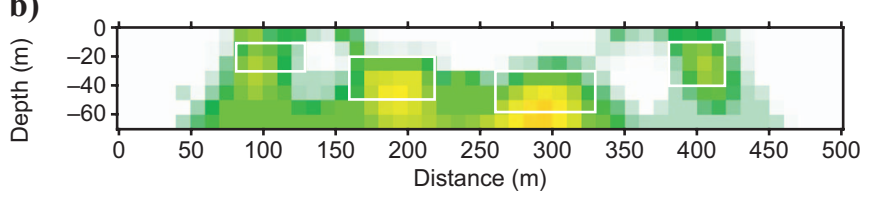

d)

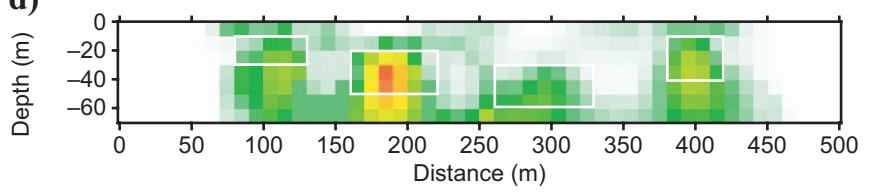

f)
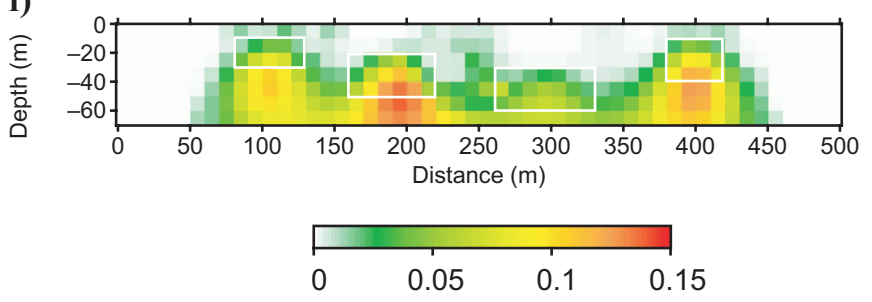

Susceptibility (SI)

Figure 10. Resistivity (left) and susceptibility (right) models obtained from inverting three airborne EM data sets for the model shown in Figure 8. The top and middle panels are for the cases in which the HCP data are collected at heights of 30 and $20 \mathrm{~m}$, respectively. The bottom panels are for the case in which both data sets are used. 
$10 \mathrm{ohm}-\mathrm{m}$ and susceptibility of 0.1 (SI units). To simulate Slingram surveys, we assume that the transmitter and receiver loops are separated by $50 \mathrm{~m}$ and maintain a common height of $1 \mathrm{~m}$. Data are collected along a 500-m line at 46 locations at intervals of $10 \mathrm{~m}$. The frequencies used in this experiment are 2500, 5000, 10,000, and $20,000 \mathrm{~Hz}$. Figure 12 shows the HCP, VCP, and VCX responses for the model in Figure 11 and the corresponding nonsusceptible model. These responses show different signatures of the conductive body for different loop configurations. The HCP configuration gives the most complicated responses, showing two pronounced troughs over the shallowest parts of the body (see the leftmost column of Figure 12). The VCP and VCX responses (in the central and rightmost columns of Figure 12, respectively) show two peaks over nearly the same locations. On the other hand, the susceptibility effects on the in-phase responses are not as clear as when using the small-loop and airborne EM methods. The differences due to the susceptibility

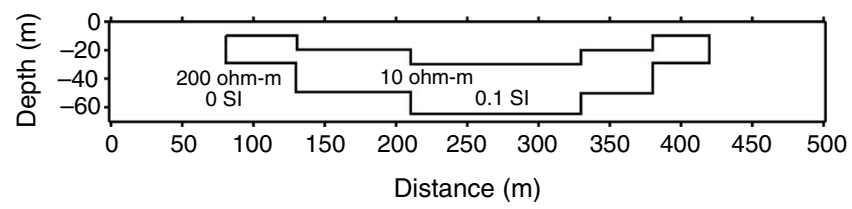

anomaly, the maximum value of which is 0.027 for the $\mathrm{HCP}$ configuration, are not significant for the most part when considering the data error associated with the possible misalignment of the loop-loop geometry

In the inversions, the simulated responses were contaminated with Gaussian noise, with a standard deviation of 0.01 , or $1 \%$ of the free-space field. The forward modeling was performed on a $69 \times 29$ $\times 34$ grid. The starting model was a half-space of 100 -ohm-m resistivity and $10^{-4}$ (SI units) susceptibility. The parameters $\lambda$ and $\alpha$ used were the same as those in the previous examples. The inversion results for the HCP data alone, the $\mathrm{HCP}$ and VCP data together, and all three data sets combined are shown in Figure 13. The data misfits for the three cases are 0.01. Although the resistivity is well recovered particularly when the data from different configurations are combined, none of the inversions is successful in recovering the susceptibility. We further examined the performance of the simultaneous inversion by adding data obtained with the 20-m loop separation and data obtained at $500 \mathrm{~Hz}$ but found no significant improvement in the susceptibility model. From this experiment we can say that the susceptibility effect in the Slingram method is practically negligible compared with those in the small-loop and airborne EM methods.

Figure 11.2D model used to generate the synthetic data for Slingram EM surveys.
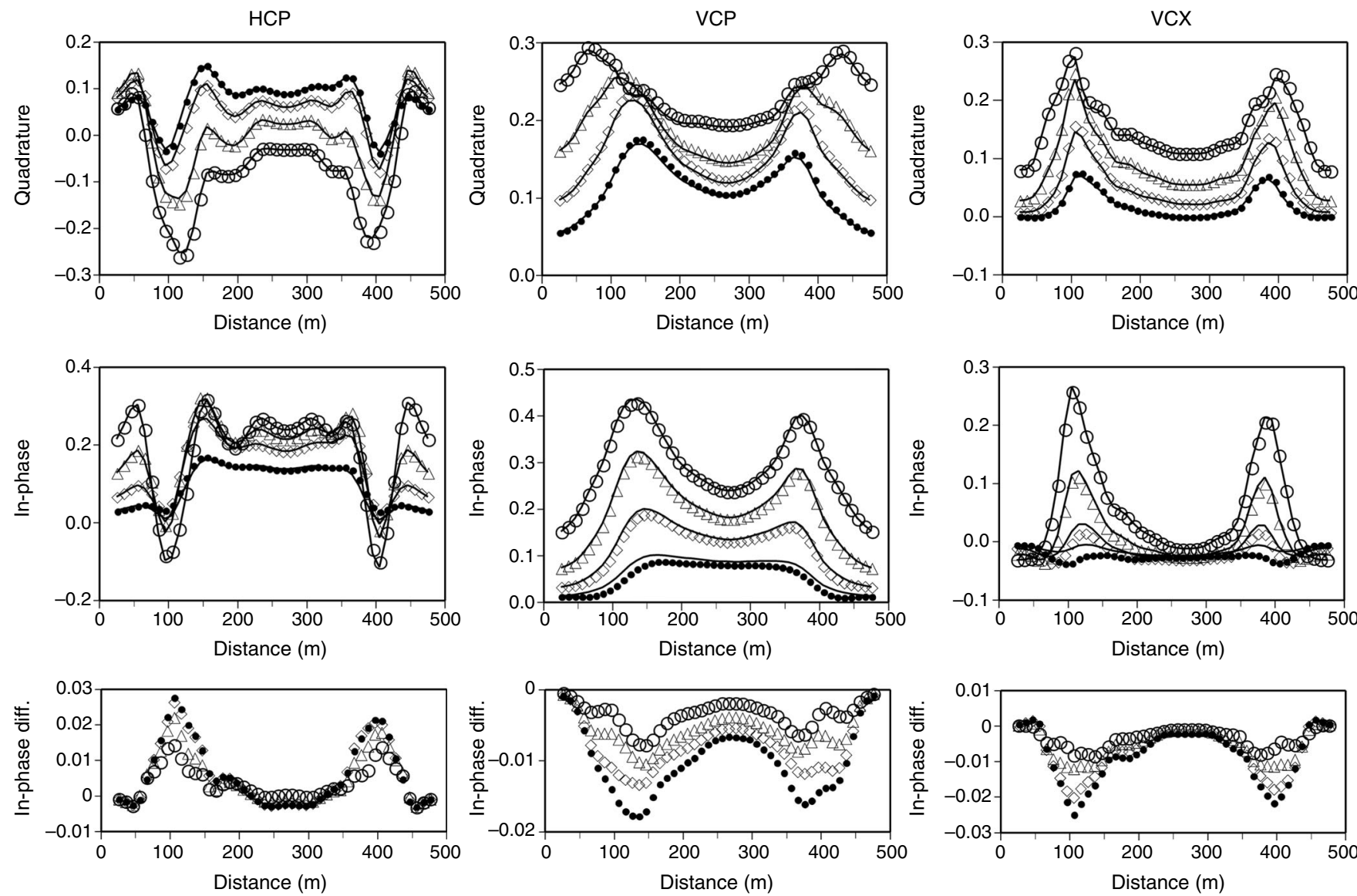

Figure 12. The Slingram EM responses for the HCP (left), VCP (center), and VCX (right) configurations calculated for the model shown in Figure 11 (symbols) and the same model without susceptibility anomalies (solid lines). The top and middle panels show the quadrature and in-phase components, respectively. The bottom panels show the differences in the in-phase responses between the models with and without susceptibility anomalies. The frequencies used are $20,000 \mathrm{~Hz}$ (open circles), $10,000 \mathrm{~Hz}$ (triangles), $5000 \mathrm{~Hz}$ (diamonds), and $2500 \mathrm{~Hz}$ (solid circles). 
a)

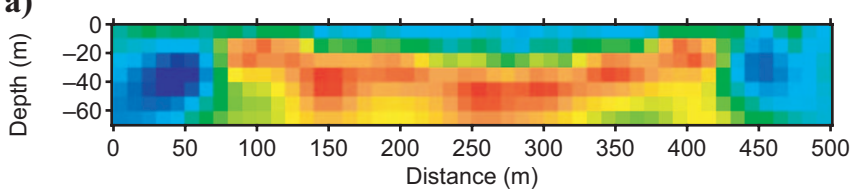

c)

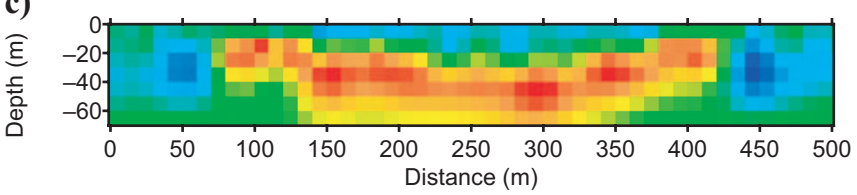

e)
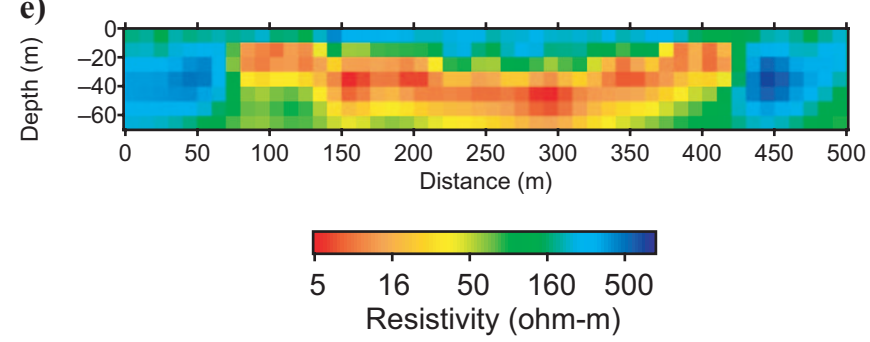

b)

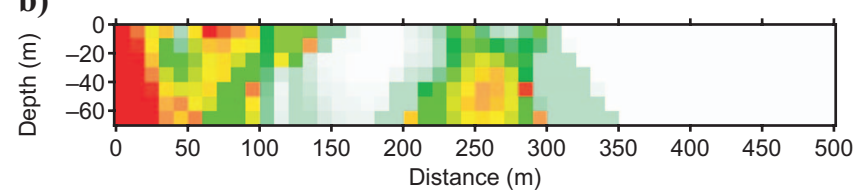

d)

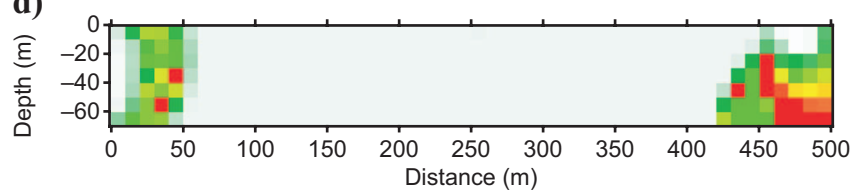

f)
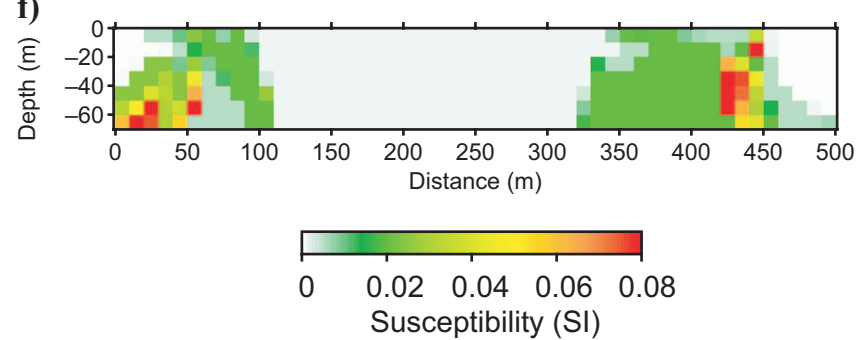

Figure 13. Resistivity (left) and susceptibility (right) models obtained from inverting three Slingram EM data sets for the model shown in Figure 11. The top, middle, and bottom panels are for the cases in which data are collected with the HCP configuration alone; the HCP and VCP configurations; and the HCP, VCP, and VCX configurations, respectively.

\section{CONCLUSIONS}

We developed a multidimensional inversion algorithm that enables the simultaneous construction of resistivity and susceptibility models from the frequency-domain loop-loop EM data. The sensitivities of the loop-loop EM responses with respect to resistivity and susceptibility can be obtained from the FD modeling results by assuming a magnetic dipole source at the receiver location.

The simultaneous inversion of small-loop EM data collected at a single height is shown to be unstable and likely to produce unreliable susceptibility models, although the resistivity can be recovered well regardless of the relative weights of the two parts of the model constraints. This is because the effect of susceptibility on the in-phase response is nearly static for the given frequency range. Thus, if the data are provided for different loop-loop geometries in addition to multiple frequencies, the simultaneous inversion can construct resistivity and susceptibility well. An additional benefit of collecting data from multiple heights and/or multiple loop configurations is that the offset errors inherent in the small-loop EM data can be removed by incorporating them into the simultaneous inversion as unknown parameters. Collecting the data from multiple heights is also useful in airborne EM surveys to improve the reliability of the resistivity and susceptibility models. Among the loop-loop EM methods discussed here, the Slingram method is relatively insensitive to susceptibility anomalies and thus is not suited to recovering the susceptibility distribution from inversion even if data are obtained with different loop configurations.

\section{ACKNOWLEDGMENTS}

The Basic Research Project of the Korea Institute of Geoscience and Mineral Resources funded by the Ministry of Knowledge Economy of Korea and a Grant-in-Aid for Scientific Research (19560812) from the Japan Society for the Promotion of Science supported this work. We thank Souvik Mukherjee, the anonymous reviewers, and the associate editor (Mark Everett) for their constructive comments that improved the clarity of the paper.

\section{REFERENCES}

Alumbaugh, D. L., G. A. Newman, L. Prevost, and J. N. Shadid, 1996, Threedimensional wideband electromagnetic modeling on massively parallel computers: Radio Science, 31, 1-23, doi: 10.1029/95RS02815.

Beard, L. P., and J. E. Nyquist, 1998, Simultaneous inversion of airborne electromagnetic data for resistivity and magnetic permeability: Geophysics, 63, 1556-1564, doi: 10.1190/1.1444452.

Bjorck, A., 1996, Numerical methods for least squares problem: Society for Industrial and Applied Mathematics.

Chave, A. D., S. C. Constable, and R. N. Edwards, 1991, Electrical exploration methods for the seafloor, in M. N. Nabighian, ed., Electromagnetic methods in applied geophysics, volume 2: SEG, 931-966.

Farquharson, C. G., D. W. Oldenburg, and P. S. Routh, 2003, Simultaneous 1D inversion of loop-loop electromagnetic data for magnetic susceptibility and electrical conductivity: Geophysics, 68, 1857-1869, doi: 10.1190/ 1.1635038.

Frischknecht, F. C., V. F. Labson, B. R. Spies, and W. L. Anderson, 1991, Profiling methods using small sources, in M. N. Nabighian, ed., Electromagnetic methods in applied geophysics, volume 2: SEG, 105-270.

Hansen, R. O., L. Racic, and V. J. S. Grauch, 2005, Magnetic methods in near-surface geophysics, in D. K. Butler, ed., Near-surface geophysics: SEG, 151-175.

Huang, H., and D. C. Fraser, 2000, Airborne resistivity and susceptibility mapping in magnetically polarizable areas: Geophysics, 65, 502-511, doi: 10.1190/1.1444744.

Huang, H., and D. C. Fraser, 2003, Inversion of helicopter electromagnetic data to a magnetic conductive layered earth: Geophysics, 68, 1211-1223, doi: 10.1190/1.1598113.

Mackie, R. L., J. T. Smith, and T. R. Madden, 1994, Three-dimensional electromagnetic modeling using finite difference equations: The magnetotelluric example: Radio Science, 29, 923-935, doi: 10.1029/94RS00326.

McGillivray, P. R., D. W. Oldenburg, R. G. Ellis, and T. M. Habashy, 1994, Calculation of sensitivities for the frequency-domain electromagnetic problem: Geophysical Journal International, 116, 1-4, doi: 10.1111/ j.1365-246X.1994.tb02121.x.

Palacky, G. J., and G. F. West, 1991, Airborne electromagnetic methods, in M. N. Nabighian, ed., Electromagnetic methods in applied geophysics, volume 2: SEG, 811-879. 
Reid, J. E., A. Pfaffling, and J. Vrbancich, 2006, Airborne electromagnetic footprints in 1D earth: Geophysics, 71, no. 2, G63-G72, doi: 10.1190/ 1.2187756 .

Sasaki, Y., 2001, Full 3-D inversion of electromagnetic data on PC: Journal of Applied Geophysics, 46, 45-54, doi: 10.1016/S0926-9851(00)000380 .

Sasaki, Y., J.-S. Son, C. Kim, and J.-H. Kim, 2008, Resistivity and offset error estimations for the small-loop electromagnetic method: Geophysics, 73, no. 3, F91-F95, doi: 10.1190/1.2842044

Smith, J. T., 1996, Conservative modeling of 3-D electromagnetic fields: Part II. Biconjugate gradient solution and an accelerator: Geophysics, 61 1319-1324, doi: 10.1190/1.1444055.

Tripp, A. C., G. W. Hohmann, and C. M. Swift Jr., 1984, Two-dimensional resistivity inversion: Geophysics, 49, 1708-1717, doi: 10.1190/1.1441578.
Weidelt, P., 1975, Inversions of two-dimensional conductivity structure: Physics of the Earth and Planetary Interiors, 10, 282-291, doi: 10.1016/ 0031-9201(75)90054-0.

Won, I. J., 2003, Small frequency-domain electromagnetic induction sensors: The Leading Edge, 22, 320-322, doi: 10.1190/1.1572084.

Won, I. J., and H. Huang, 2004, Magnetometers and electro-magnetometers: The Leading Edge, 23, 448-451, doi: 10.1190/1.1756834.

Yee, K. S., 1966, Numerical solution of initial boundary problems involving Maxwell's equations in isotropic media: IEEE Transactions on Antennas and Propagation, 14, 302-307, doi: 10.1109/TAP.1966.1138693.

Zhang, Z., and D. W. Oldenburg, 1999, Simultaneous reconstruction of 1-D susceptibility and conductivity from electromagnetic data: Geophysics, 64,33-47, doi: 10.1190/1.1444528. 